\title{
Flora vascular y formaciones vegetacionales en el sitio de estudios socio-ecológicos a largo plazo, isla Gonzalo, archipiélago Diego Ramírez (56 $31^{\circ}$ 'S), Chile
}

\author{
Vascular flora and vegetational types at the long-term socio-ecological studies site, \\ Gonzalo Island, Diego Ramírez Archipelago (56³1'S), Chile
}

Roy Mackenzie ${ }^{1,4}$, Osvaldo Vidal ${ }^{2}$, Sebastián Rosenfeld ${ }^{3}$, Tamara Contador ${ }^{1,4}$, Omar Barroso ${ }^{1}$, Bernard Goffinet ${ }^{1,5}$, Francisca Massardo ${ }^{1}$, Patricio Arce-Johnson ${ }^{7}$ \& Ricardo Rozzi ${ }^{1,6}$

\section{Resumen}

La red de sitios de estudios socio-ecológicos a largo plazo en la Reserva de la Biósfera Cabo de Hornos (LTSER-Cabo de Hornos) se extiende hoy hasta la isla Gonzalo (56³1'S), archipiélago Diego Ramírez, como su sitio más austral. Ésta es también la isla más meridional del continente americano y de la ecorregión subantártica de Magallanes, albergando una enorme diversidad de fauna marina que fue intensamente explotada luego de su descubrimiento, hace 400 años. El objetivo general de la red LTSERCabo de Hornos es detectar impactos del cambio socio-ambiental global y contribuir a su mitigación. El objetivo específico de este trabajo es proveer una descripción actualizada de la flora vascular presente en la isla Gonzalo, que es un sitio de monitoreo crítico para el nuevo Parque Marino Islas Diego Ramírez-Paso Drake. El archipiélago Diego Ramírez forma parte de la región fitogeográfica subantártica, albergando formaciones vegetacionales únicas en el territorio chileno. Se actualiza la lista de especies de plantas vasculares registradas en la isla Gonzalo, la única habitada del archipiélago. El registro incluye ocho especies de plantas vasculares que comprenden la totalidad de especies previamente registradas en los islotes y las tres islas mayores del archipiélago (Gonzalo, Bartolomé y Norte). Ninguna de las especies colectadas tiene problemas de conservación. El muestreo confirmó que en la isla Gonzalo no ha ocurrido establecimiento de flora vascular exótica, incluso en lugares intervenidos alrededor del Faro de la Armada de Chile. Se recomienda un monitoreo estricto para prevenir el establecimiento y diseminación de plantas exóticas que potencialmente podrían alterar la vegetación de este archipiélago.

\section{Palabras clave:}

Diego Ramírez, especies exóticas, ecorregión subantártica de Magallanes, islas subantárticas, parque marino.

1 Programa de Conservación Biocultural Subantártica, Instituto de Ecología y Biodiversidad (IEB-Chile), Universidad de Magallanes,

Puerto Williams, Chile.

https://orcid.org/0000-0001-6620-1532

$₫$ roy.mackenzie@umag.cl

2 Laboratorio de Botánica,

Universidad de Magallanes,

Avenida Bulnes 01890, Punta Arenas, Chile.

3 Laboratorio de Ecosistemas Marinos Antárticos y Subantárticos (LEMAS), Universidad de Magallanes, Punta Arenas, Chile.

4 Laboratorio Wankara de Ecosistemas Dulceacuícolas Subantárticos y Antárticos, Universidad de Magallanes \& Instituto de Ecología y Biodiversidad (IEB).

Puerto Williams, Chile.

5 Department of Ecology and Evolutionary Biology, University of Connecticut, EE.UU..

6 Subantarctic Biocultural Conservation Program,

Department of Philosophy and Religion \&

Department of Biological Sciences,

University of North Texas, EE.UU.

7 Facultad de Ciencias Biológicas,

Pontificia Universidad Católica de Chile. 


\section{Abstract}

The network of Long-term Socio-ecological Research sites within the Cape Horn Biosphere Reserve (LTSER-Cape Horn) extends today to the Gonzalo Island (56 $\left.31^{\prime} \mathrm{S}\right)$, Diego Ramírez Archipelago, as its southernmost site. This is also the southernmost island of the American continent and of the sub-Antarctic ecoregion of Magallanes, housing a huge diversity of marine fauna that was intensely exploited after its discovery, exactly 400 years ago. The general objective of the LTSER-Cape Horn network is to detect the impacts of global socio-environmental change and contribute to their mitigation. The specific objective of this work is to provide an updated description of the vascular flora present on Gonzalo Island, a critical monitoring site for the new Diego Ramírez Islands-Paso Drake Marine Park. The Diego Ramírez Archipelago is part of the sub-Antarctic phytogeographic region, harboring vegetation formations that are unique in the Chilean territory. Our work updated the list of vascular plant species of Gonzalo Island, the only one that is permanently inhabited. The records include eight species of vascular plants that comprise all the species of vascular plants previously recorded in the islets as well as in the three major islands of the archipelago (Gonzalo, Bartolomé and North). None of the eight collected species has conservation problems. Our sampling confirmed that there has been no establishment of exotic vascular flora in Gonzalo Island. Even places that have been disturbed near the base maintained by the Chilean Navy had no presence of exotic species. Strict monitoring is recommended to prevent the establishment and dissemination of exotic plants that could potentially arrive on this island, and the Diego Ramírez Archipelago.

\section{Key words:}

Diego Ramírez, exotic species, sub-Antarctic ecoregion of Magallanes, sub-Antarctic islands, marine park.

\section{INTRODUCCIÓN}

Las islas Diego Ramírez (56²9'S; 6844'O) se ubican aproximadamente a $111 \mathrm{~km}$ al sudoeste del Cabo de Hornos, y son las únicas superficies sobre el nivel del mar incluidas en el Parque Marino
Islas Diego Ramírez-Paso Drake, de 140.000 km² de extensión (Rozzi et al. 2017). Este archipiélago comprende un conjunto de islotes y tres islas mayores: Gonzalo, Bartolomé y Norte, separadas en dos grupos y de las cuales sólo la más meridional (Gonzalo) está permanentemente habitada por un reducido número de funcionarios de la Armada de Chile.

El archipiélago Diego Ramírez ha sido visitado por distintos exploradores europeos y de otras regiones desde que en 1619 fuera descubierto por la expedición española al mando del capitán Bartolomé García de Nodal (Martinic, 2005, 2018). Este archipiélago posee una especial valoración como patrimonio tanto cultural como natural debido a su estratégica posición geográfica en el extremo austral de la plataforma continental americana (Rozzi et al. 2017) y su excepcional biodiversidad marina. De acuerdo a lo recopilado por el historiador Mateo Martinic (2018), estas islas eran blanco frecuente de cazadores furtivos en barcos de bandera extranjera, que explotaban las abundantes colonias de mamíferos marinos como focas, elefantes y lobos marinos, especialmente el lobo de dos pelos (Arctocephalus australis Zimmermann 1783). La especial demanda mundial de cueros y pieles finas que hubo en el siglo XIX, así como también de aceite y grasa de ballena y otros mamíferos marinos, justificó una explotación irracional en estas regiones, llevando a estas especies cerca de su extinción. Recién en la década de 1840 el agotamiento y migración de las especies comerciales disminuyó notoriamente las actividades de caza y desde entonces las islas fueron ignoradas. Posteriormente, el contraalmirante Donald McIntyre Griffiths vio en estas islas un apostadero estratégico para la presencia soberana activa de la República de Chile en los mares del sur, y en 1953 se instaló un destacamento naval (Puesto de Vigía y Señales, PVS) en la isla Gonzalo. Este destacamento está habitado permanentemente y dotado de radiocomunicaciones y registro meteorológico, permitiendo el control de la navegación interoceánica, el auxilio a los eventuales naufragios y la mantención del control de la caza furtiva de especies marinas en el maritorio nacional. Esta protección geopolítica permitió la recuperación de parte de las poblaciones marinas hasta las 
proporciones actuales, detalladas en el Informe Técnico para el Parque Marino recientemente inaugurado (Rozzi et al. 2017).

El reconocimiento y estudio del patrimonio natural del archipiélago Diego Ramírez comienza con el geólogo E. Aubert de la Rue, quien fuera el primero en realizar una visita científica en 1953. No habría una segunda hasta 1972, cuando el botánico Edmundo Pisano, del Instituto de la Patagonia, realizara una revalorización de las islas como patrimonio natural singular y diferente de lo que se conocía hasta entonces (Pisano, 1972).

Por estas dos razones, el archipiélago Diego Ramírez representa hoy un sitio idóneo para el monitoreo de la biodiversidad subantártica y sus respuestas al cambio socio-ambiental global.

Las islas Diego Ramírez tienen características vegetacionales que son propias de las islas subantárticas. La flora tiene una marcada similitud con aquella de otras islas de la ecorregión subantártica de Magallanes (sensu Rozzi et al. 2012), en particular con la isla Hornos (cfr. Dollenz, 1980; Pisano, 1980a, b), en menor grado con la flora de las islas Malvinas (Moore, 1968; Pisano \& Schlatter, 1981a; Schlatter \& Riveros, 1987) y en el continente antártico (Moore, 1968). La flora de las islas subantárticas está caracterizada principalmente por la presencia de gramíneas coriáceas formadoras de tussock (pastos altos) y la ausencia de especies leñosas (Fuller, 1914; Moore, 1968). Esta formación vegetacional fue reconocida por Pisano y Schlatter (1981b) como una provincia fitogeográfica diferente, denominada gramíneoturbosa subantártica.

Nuestro trabajo tiene como objetivo reportar un inventario florístico actualizado de la flora vascular en el sitio de estudios socio-ecológicos a largo plazo en la isla Gonzalo, que constituye un sitio de monitoreo terrestre para el nuevo Parque Marino Islas Diego Ramírez-Paso Drake (Rozzi et al. 2017). Este artículo proveerá una línea de base fundamental y los primeros indicadores de biodiversidad local (sensu Noss, 1990) para el futuro monitoreo ecológico de este particular ecosistema terrestre en el archipiélago. Nuestro artículo se centra en la flora vascular y complementa el estudio de la flora briofítica (no-vascular) para este sitio desarrollado por Goffinet et al. (2020, este volumen).

\section{MATERIALES Y MÉTODOS}

Este catálogo florístico fue confeccionado sobre la base de colectas realizadas en noviembre de 2016, en junio de 2017 y en abril de 2018 en la isla Gonzalo y depositadas en el Herbario del Instituto de la Patagonia (HIP), donde se encuentran además los especímenes de herbarios (o exsiccata) depositados en distintos años por Pisano (1972), Schlatter (1981) y Herrera y Johnson (1984) luego de sus respectivas visitas a las islas.

\section{Fertilidad de la turba}

Se realizó un análisis químico de fertilidad del histosol producido por Poa flabellata para caracterizar los atributos biogeoquímicos del suelo superficial que son relevantes para el crecimiento vegetal. Para ello, en diciembre de 2017 se recolectaron aproximadamente $500 \mathrm{~g}$ de turba desde el suelo alrededor de una champa de Poa flabellata, y conservada en frío $\left(4-8^{\circ} \mathrm{C}\right)$ en una bolsa plástica hermética hasta su análisis en laboratorio. La muestra fue enviada al Laboratorio de Análisis Agrícola Agrolab Ltda. (Santiago de Chile) en enero de 2018. Los métodos de los análisis químicos realizados a la muestra de suelo se encuentran detallados en Pearcy et al. (1989). Brevemente, se obtuvieron los valores de $\mathrm{pH}$ (en agua, relación 1:2,5), conductividad eléctrica (por el método de extracto saturado), materia orgánica (método Walkley \& Black) y concentración de macronutrientes nitrógeno, fósforo y potasio disponibles (métodos de Bremmer, de Olsen y de acetato de amonio, respectivamente).

La nomenclatura científica utilizada para los especímenes vegetales sigue a Rodríguez et al. (2018), mientras que el estado de conservación de las especies se determinó siguiendo el "Inventario Nacional de Especies de Chile" del Ministerio del Medio Ambiente. Este inventario clasifica especies nativas en categorías de conservación según los criterios definidos por UICN (http://especies.mma.gob.cl/CNMWeb/Web/ WebCiudadana/Default.aspx).

\section{RESULTADOS}

Para la isla Gonzalo se han registrado previamente ocho especies de plantas vasculares (Pisano, 1972; Pisano \& Schlatter, 1981a): Poa flabellata (Lam.) Raspail, Cardamine glacialis 
(G. Forst.) DC., Callitriche antarctica Engelm ex Hegelm., Crassula moschata G. Forst., Plantago barbata G. Forst., Colobanthus quitensis (Kunth) Bartl., Ranunculus biternatus Sm. y Leptinella scariosa Cass. Esta lista incluye todas las especies registradas hasta el momento en las tres islas mayores e islotes del archipiélago (Pisano \& Schlatter, 1981a).

A la fecha, ninguna de estas especies de plantas vasculares ha sido clasificada en un estado de conservación según el Reglamento de Clasificación de Especies del Ministerio del Medio Ambiente, pero por sus amplias distribuciones deberían ser clasificadas como de Preocupación Menor (Squeo et al. 2010).

A continuación, se presenta un catálogo de las plantas vasculares registradas en la isla Gonzalo, además de algunas observaciones sobre el hábitat y ecología de cada especie.

\section{1 y 2: Poa flabellata (Lam.) Raspail (Gramineae) y Crassula moschata G. Forst. (Crassulaceae)}

Poa flabellata, conocida comúnmente como coirón, pasto alto o tussock (en inglés), es la gramínea coriácea dominante en las islas Diego Ramirez (Fig. 1A). En los parches donde domina alcanza una densa cobertura continua (Figs. 1B y 1C), que varía entre un $80 \%$ y $100 \%$ (Pisano \& Schlatter, 1981b). En aquellos lugares donde la cobertura ha sido perturbada y la luz penetra el dosel (e.g. nidos abandonados), o creciendo cespitosamente sobre rocas semienterradas, se encuentra también en pequeña proporción Crassula moschata. Esta planta suculenta crece de manera reptante, con hojas y tallo verdes (Fig. 1D). En zonas completamente expuestas a la luz solar su tallo adquiere un tono rojizo y sus hojas pueden ser amarillas o verde claro. P. flabellata y C. moschata forman parte de la asociación fitosociológica Poetum flabellatae, vegetación característica de las islas subantárticas de esta región (Pisano \& Schlatter, 1981b).

Poa flabellata habitualmente supera el metro y medio de alto y alcanza hasta 2 metros de altura en la isla Gonzalo, donde genera un ambiente protegido del viento. En este hábitat la variación térmica diaria en primavera es de sólo $5.4^{\circ} \mathrm{C}$, casi la mitad de la variación térmica fuera de las formaciones de gramíneas (Contador et al. 2020, en este volumen). Esto provee un "refugio microclimático" bajo las champas, donde las formaciones densas de gramíneas facilitan el desarrollo de colonias nidificantes de aves marinas como el pingüino de Magallanes (Spheniscus magellanicus Forster 1781), el pingüino de penacho amarillo (Eudyptes chrysocome Forster 1781), el pingüino macaroni (Eudyptes chysolophus Brandt 1837), el petrel azulado (Halobaena caerulea Gmelin 1789), el yunco de los canales (Pelecanoides urinatrix coppingeri Mathews 1912), el albatros de ceja negra (Thalassarche melanophrys Temminck 1828) y el albatros de cabeza gris (Thalassarche chrysostoma Forster 1785), entre otras aves marinas y terrestres (Robertson et al. 2007; Rozzi et al. 2017). Estas aves pueden llegar a conformar una biomasa superior a las 1.000 toneladas durante la época estival en el archipiélago Diego Ramírez (Schlatter $\&$ Riveros, 1987), por lo que realizan un aporte importante de nitrógeno y fósforo al ecosistema en forma de guano, como se pudo constatar en la vegetación aledaña a los nidos y a los polluelos.

Las colonias de albatros y pingüinos se distribuyen hasta las zonas más altas de la isla Gonzalo (130 msnm), en donde crean numerosos pasadizos a través de las formaciones de $P$. flabellata. Estos pasadizos pueden tener hasta 100 centímetros de ancho. Estas colonias de aves utilizan la turba y el pasto alto para su nidificación. En las zonas bajas de la isla, colonias de lobo de dos pelos (Arctocephalus australis) y de elefante marino (Mirounga leonina Linnaeus 1758) compactan la turba y generan una costra impermeable en la superficie del suelo, que podría dificultar la regeneración del coirón.

Las colonias de albatros de ceja negra presentan un patrón notable, puesto que mantienen activamente claros fangosos, planos y despejados de Poa flabellata, aquéllas quedan guarecidas en las partes bajas de los relieves que provee la geomorfología de la isla Gonzalo. El petrel azulado y el yunco de los canales tienen hábitos nocturnos y construyen madrigueras en el suelo turboso que le permiten refugiarse durante el día, éstas pueden tener hasta $70 \mathrm{~cm}$ de profundidad (Pisano \& Schlatter, 1981b). En estos sitios, las deyecciones de las aves crean una turba rica en nutrientes que aumenta la fertilidad del suelo y fomenta el crecimiento de las plantas. 
La determinación de la fertilidad de la turba de Poa flabellata en la isla Gonzalo se determinó en un único punto, cercano a un nido activo de albatros. La turba se encontraba permanentemente húmeda y mantenía una rica diversidad de invertebrados asociados a las raíces del coirón (Contador et al. 2020 , en este volumen), con un porcentaje de materia orgánica de 52,8\%. Se determinó un pH de 3,8, definido por la FAO como extremadamente ácido y restrictivo para las plantas (http://www.fao.org/ soils-portal/en/). Este factor, sumado a un sustrato anaeróbico y saturado de agua, inhibe la actividad microbiana y fomenta la acumulación de la materia vegetal en forma de turba. Las concentraciones de $\mathrm{N}, \mathrm{P}$ y K superaron los $1000 \mathrm{mg} / \mathrm{kg}$ (N disponible: $1373 \mathrm{mg} \mathrm{kg}{ }^{-1}$, P disponible: $1049 \mathrm{mg} \mathrm{kg}^{-1}$, K disponible: $1837 \mathrm{mg} \mathrm{kg}^{-1}$ ). Estos altos valores se asociarían a la acumulación de guano y reciclaje de los macronutrientes biodisponibles para las plantas. Finalmente, la alta concentración de sales electrolíticas determinada en la muestra clasifica a esta turba como muy salina, debido a la influencia del ambiente marino sobre el suelo, atributo que afectaría la composición de las comunidades vegetales en este ecosistema subantártico.

3 y 4: Cardamine glacialis (G. Forst.) DC. (Brassicaceae) y Callitriche antarctica Engelm ex Hegelm. (Plantaginaceae).

Ambas especies se desarrollan bajo el dosel de P. flabellata así como en las orillas de cursos de agua, en espacios más abiertos (Figs. 1E y 1F), con un crecimiento en este último caso de mayor vigor. Estas dos especies forman parches de plantas ocupando extensiones de varios metros sobre el terreno húmedo (Fig. 1G). Son especialmente abundantes en zonas intervenidas alrededor de la Estación de la Armada o en la turba expuesta en el sendero que comunica con la baliza.

\section{5 y 6: Plantago barbata G. Forst.}

(Plantaginaceae) y Colobanthus quitensis

(Kunth) Bartl. (Caryophyllaceae).

Estas plantas suculentas forman densas sinusias pulvinadas, creando un tapiz pantanosoturboso asociado a terrenos anegados, sensibles a las pisadas en explanadas que se extienden por varios metros. Plantago barbata y C. quitensis tienen, en general, un tipo de crecimiento en carpeta y tamaños similares (Figs. $1 \mathrm{H}$ y $1 \mathrm{I}$ ), de aproximadamente 10-15 centímetros de altura y ancho variable (hasta 50 centímetros). Asociados a estas dos especies de plantas vasculares, se registraron en medio del tapiz vegetal parches verde oscuro del musgo Hennediella heimii (Hedw.) R.H. Zander (Goffinet et al. 2020, en este volumen). No se observaron musgos esfagnosos.

En la isla Gonzalo se extiende una notoria meseta plana de aproximadamente $900 \mathrm{~m}^{2}$ y de $90 \mathrm{~m}$ de altitud dominada por esta vegetación, a pocos metros al oeste del faro. Pisano y Schlatter (1981b) definieron esta asociación fitosociológica, que se da principalmente en lugares intervenidos, como Plantago-Colobanthetum quitensei (Fig. $1 \mathrm{~J})$. No se observó crecimiento de Poa flabellata en este sustrato. Entre ambos tipos vegetacionales se registró un límite muy definido, excepto algunos pequeños parches de $P$. flabellata en los márgenes del tapiz de Plantago. No se observaron aves anidando en este tipo vegetal, con excepción de algunos albatros que construyeron sus nidos en el límite donde comienzan las formaciones de coirón.

$\mathrm{El}$ origen de esta formación vegetal en ese lugar es incierto. Pisanoy Schlatter (1981b) destacan la ocurrencia puntual de este tipo vegetacional en la isla Gonzalo, puesto que no fue observada en otras partes de la isla ni del archipiélago Diego Ramírez. Ellos discuten la posibilidad de que la actividad de una antigua colonia de pingüinos haya labrado esta curiosa formación vegetal. Alternativamente, esta formación podría tener un origen antrópico debido a que en este lugar se encuentran restos de un cerco alambrado que mantenía confinado un pequeño grupo de ganado ovino, el cual consumía los brotes de coirón (comunicación personal J. Mella, dotación del faro Diego Ramírez).

7 y 8: Ranunculus biternatus Sm.

(Ranunculaceae) y Leptinella scariosa Cass. (Asteraceae)

Estas dos pequeñas herbáceas poseen un crecimiento rastrero y se encontraron sólo en terrenos intervenidos (Fig. 1K), en los alrededores de la Estación de la Armada y formando sinusias herbáceas (Figs. 1L y 1M). En los extremos este 


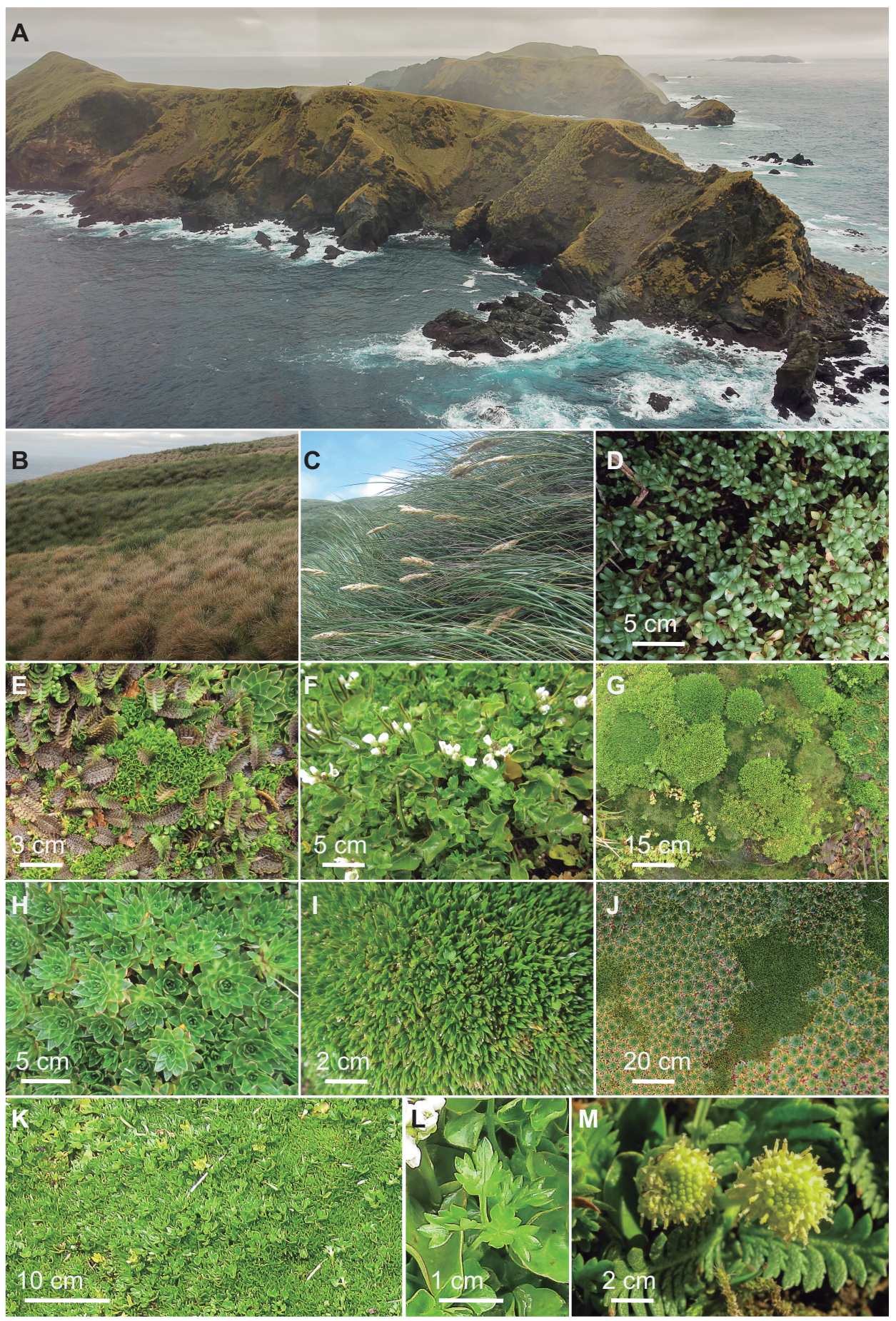

Fig. 1. Catálogo de imágenes de las plantas vasculares presentes en las islas Diego Ramírez. A) Vista aérea de la isla Gonzalo. Detrás, se observa la isla Bartolomé y al fondo, la isla Norte; archipiélago Diego Ramírez. B) Tussock o pastos altos de Poa flabellata, C) espiguillas de P. flabellata, D) Crassula moschata, E) Callitriche antarctica,

F) Cardamine glacialis con flor y silicuas, G) Parches de C. antarctica sobre turba, H) Plantago barbata,

I) Colobanthus quitensis, J) tapiz de P. barbata y C. quitensis, K) tapiz de Ranunculus biternatus y C. quitensis,

L) Ranunculus biternatus, M) Leptinella scariosa. Fotografías de Omar Barroso, Roy Mackenzie y Bernard Goffinet. 
y oeste de la isla Gonzalo, menos perturbados, no se observó la presencia de estas plantas. Pisano y Schlatter (1981a) tampoco registraron estas especies en las otras islas del archipiélago, $y$ proponen que ambas habrian llegado a la isla Gonzalo por transporte humano y se habrian establecido en los terrenos alterados por las construcciones de la baliza y la Estación de la Armada construida el año 1956.

\section{DISCUSIÓN Y CONCLUSIONES}

En contraste con las demás islas y ecosistemas terrestres de la ecorregión subantártica de Magallanes, caracterizados por la presencia de bosques siempreverdes dominados por el coigüe de Magallanes (Nothofagus betuloides [Mirb.] Oerst 1871) (Rozzi et al. 2006), el archipiélago Diego Ramírez carece de especies leñosas. El inventario florístico dado a conocer en este trabajo presenta una actualización del estudio realizado por Pisano (1972) y revisado, posteriormente, por Pisano y Schlatter (1981a, b). A pesar de los eventos de zoocoría provocados por el movimiento migratorio de aves y el impacto de los seres humanos en mayor medida por las intervenciones al terreno que fueron indispensables para la instalación permanente de la dotación de la Armada de Chile en la isla Gonzalo, no se encontraron especies de plantas exóticas.

Las dos asociaciones vegetales principales determinadas por Pisano y Schlatter (1981b): Plantago-Colobanthetum quitensei y Poetum flabellatae, siguen siendo las únicas con cobertura destacable, probablemente debido a su tolerancia a la salinidad oceánica y capacidad de aprovechamiento del exceso de los macronutrientes nitrógeno, fósforo y potasio proveniente del guano de las aves marinas.

La ausencia de plantas exóticas representa uno de los resultados más relevantes de nuestro estudio. Existen muy pocas áreas en el planeta que tienen la característica de estar libres de especies exóticas (Drake et al. 1989; Vitousek, 1994; Vitousek et al. 1996; Primack et al. 2001) y especialmente muy pocas islas subantárticas están libres de especies exóticas (Gremmen \& Smith, 1999; McIntosh \& Walton, 2000). Por lo tanto, esta condición constituye un atributo singular y de la mayor importancia para la conservación de la biodiversidad en la unidad geográfica más austral del continente americano.

A tan sólo 100 kilómetros al noreste de las islas Diego Ramírez se encuentra el archipiélago Cabo de Hornos. Este grupo de islas conserva su condición de estar casi completamente libre de especies de plantas exóticas (Rozzi et al. 2004). Sólo 3 especies exóticas se registraron en el archipiélago Cabo de Hornos, presentes en sitios muy específicos y acotados: (1) Rumex crispus L. en la costa de isla Bayly, frente a la entrada este al canal Victoria; (2) Stellaria media L. en el margen de bosque de coigüe de Magallanes (Nothofagus betuloides) en el litoral de caleta Saint Martin, isla Hermite; en la costa este de isla Bandurrias bajo matorral costero de Hebe elliptica (G. Forster) Pennell y Gaultheria mucronata (L. f.) Hook. \& Arn.; (3) Poa nemoralis L. en la costa norte, punta Dillon, de isla Grevy bajo el matorral costero de H. elliptica (Rozzi et al. 2004). Los sitios de isla Grevy, isla Bayly e isla Bandurrias se encuentran en el sector donde tuvo lugar alguna actividad colonizadora a través del establecimiento de una misión anglicana en la isla Bayly. La misión incluyó la introducción de ganado caprino y ovino (Canclini, 1999). Por otro lado, en caleta Saint Martin, la presencia de Stellaria media podría estar asociada a los frecuentes desembarcos de pescadores como también de expediciones científicas y de turismo en yates privados (Rozzi et al. 2004).

Respecto a la flora del archipiélago Cabo de Hornos, Pisano (1980a, pp 179) señaló que "las condiciones ambientales prevalentes en el archipiélago pueden considerarse como limitantes para un gran grupo de especies exóticas naturalizadas en numerosas localidades del sur de Tierra del Fuego. La escasa o nula alteración de su vegetación natural por efectos antrópicos y la poca frecuencia de visitas humanas son también desfavorables para este tipo de invasiones. Llama la atención la ausencia de otras especies ubicuas en altas latitudes del hemisferio sur, algunas de las cuales alcanzan hasta las islas subantárticas".

En el archipiélago Cabo de Hornos, la presencia de especies exóticas está asociada a la actividad humana (Rozzi et al. 2004). Dada la cercanía entre ambos archipiélagos, es fundamental tener un monitoreo permanente de la vegetación en la isla Gonzalo que permita documentar $y$, en 
el mejor de los casos, prevenir el establecimiento de especies exóticas. Un resultado significativo de nuestro estudio es que no se ha establecido ninguna especie de planta vascular exótica en la isla Gonzalo, y que después de casi 40 años se mantiene la condición registrada por Pisano y Schlatter (1981a, b). La ocupación humana permanente en la isla Gonzalo podría favorecer el arribo de semillas y propágulos de plantas exóticas; sin embargo, sólo se ha observado el establecimiento de vegetación autóctona en las zonas intervenidas. Pisano y Schlatter (1981a) discuten la posibilidad de que Ranunculus biternatus y Leptinella scariosa hayan sido introducidas en la isla por antropocoría: $R$. biternatus se distribuye en las islas subantárticas desde el Pacífico sur hasta las islas Kerguelen, en el océano Índico (Lehnebach et al. 2017); y L. scariosa podría haber llegado a Sudamérica desde Nueva Zelanda por dispersión a larga distancia de las plantas machos y hembras (Himmelreich et al. 2012). No obstante, es necesario destacar que ambas especies sospechosas de ser exóticas tienen una amplia distribución en otras islas subantárticas (Shaw, 2005).

Del mismo modo, el clavel antártico Colobanthus quitensis, presente en todas las islas $e$ islotes del archipiélago Diego Ramírez (Pisano \& Schlatter, 1981a) y en Sudamérica, es junto a Deschampsia antarctica una de las dos únicas especies vasculares presentes en el continente antártico. Según Parkinoza et al. (2007), estas especies son reliquias migratorias que pueden haber sido dispersadas hasta allí por skúas y pingüinos en una época con un clima más favorable, lo que habría permitido su naturalización y adaptación hasta las condiciones actuales.

Un ejemplo actual de un evento de dispersión de estas características es descrito por Turner y colaboradores (2006), donde un único espécimen de la hierba subantártica Leptinella plumosa fue observado en la isla Heard, a $500 \mathrm{~km}$ de las islas Kerguelen, en un terreno rocoso expuesto luego del retroceso de un glaciar. Los autores proponen que el evento de dispersión a larga distancia de $L$. plumosa podría haber ocurrido por ornitocoría.

En resumen, el archipiélago Diego Ramírez constituiría uno de los pocos grupos de islas subantárticas libres de especies exóticas (Rozzi et al. 2017). Este estudio permitió también el inicio del monitoreo de la composición y cobertura vegetal en parcelas instaladas en la isla Gonzalo (Rozzi et al. 2020), además de las características químicas del sustrato turboso. Se inaugura así un monitoreo socio-ecológico en el ecosistema terrestre más austral de América, reconociendo su importancia biocultural para la historia de la ocupación de la región y que en enero de 2019 ha sido formalmente protegido por el nuevo Parque Marino Islas Diego Ramírez-Paso Drake (Diario Oficial 2019).

\section{AGRADECIMIENTOS}

Se agradece a la Tercera Zona Naval y a la dotación del OPV Marinero Fuentealba por las facilidades otorgadas para la participación de los investigadores en las navegaciones a las islas Diego Ramírez. También se agradece a la dotación del faro Diego Ramírez por su ayuda logística en todo momento durante las visitas de las comisiones científicas. Agradecemos el apoyo de los proyectos Pew Charitable Trust, PAI79170119 y MPG190029 de ANID y el Instituto de Ecología y Biodiversidad de Chile a través del proyecto Centros Tecnológicos de Excelencia con Financiamiento Basal ANID AFB170008. Este artículo representa una contribución del Programa de Conservación Biocultural Subantártica-Parque Etnobotánico Omora.

\section{LITERATURA CITADA}

Canclini, A. (1999). Los indios del Cabo de Hornos. Buenos Aires, Argentina: Zagier \& Urruty.

Contador, T., Rendoll, J., Mackenzie, R., Rosenfeld, S., Barroso, O., Rozzi, R., Goffinet, B., Kennedy, J., \& Convey, P. (2020). Comunidades de invertebrados terrestres del archipiélago Diego Ramírez (56³1’S), el sitio de estudios socio-ecológicos a largo plazo más austral de América: diversidad y afinidades con las islas subantárticas del océano Austral. Anales del Instituto de la Patagonia, 48,(3), 83-98.

Dollenz, O. (1980). Estudios fitosociológicos en el Archipiélago del Cabo de Hornos. Anales del Instituto de la Patagonia, 11, 191-224. 
Drake, J. A., Mooney H. A., di Castri, F., Groves, R. H., Kruger, F. J., Rejmanek, M. \& M. Williamson (eds). (1989). Biological invasions: a global perspective. John Wiley and Sons, Chichester, UK.

Fuller, G. D. (1914). Subantarctic vegetation. Botanical Gazette, 58, 96-98.

Goffinet, B., Engel, J. J., von Konrat, M., Mackenzie, R., Contador, T., Rosenfeld, S., Barroso, O., \& Rozzi, R. (2020). First bryophyte records from Diego Ramírez archipelago: changing lenses in long-term socio-ecological research at the southernmost island of the Americas. Anales del Instituto de la Patagonia, 48, en revisión.

Gremmen, N. J. M., \& Smith, Y. R. (1999). New records of alien vascular plants from Marion and Prince Edward Islands, sub-Antarctic. Polar Biology 21, 401-409.

Himmelreich, S., Breitwieser, I., \& Oberprieler, C. (2012). Phylogeny, biogeography, and evolution of sex expression in the southern hemisphere genus Leptinella (Compositae, Anthemideae). Molecular Phylogenetics and Evolution, 65(2), 464-481.

Lehnebach, C. A., Winkworth, R. C., Becker, M., Lockhart, P. J., \& Hennion, F. (2017). Around the pole: evolution of sub-Antarctic Ranunculus. Journal of Biogeography, 44(4), 875-886.

Martinic, M. (2005). Crónica de las tierras al sur del canal Beagle. Punta Arenas, Chile: Ediciones Hotel Lakutaia.

Martinic, M. (2018). Cuatro siglos del viaje de los hermanos Nodal a la Tierra del Fuego (1618-1619). Magallania, 46(2), 7-23.

McIntosh, E., \& Walton, D. W. H. (2000). Environmental management plan for South Georgia. UK: Published by British Antarctic Survey on behalf of Government of South Georgia and the South Sandwich Islands.

Moore, D. M. (1968). The vascular flora of the Falkland Islands. British Antarctic Survey Scientific Reports, 60.

Noss, R. F. (1990). Indicators for monitoring biodiversity: A hierarchical approach. Conservation Biology, 4, 355-364.

Pearcy, R. W., Ehleringer, J. R., Mooney, H. A.,
\& Rundel, P.W. (1989). Plant physiological methods. New York, US: Chapman \& Hall.

Parkinoza, I., Maidanuk, D. N., \& Kozeretska, I. A. (2007). Are Deschampsia antarctica Desv. and Colobanthus quitensis (Kunth) Bartl. migratory relicts? Cytology and Genetics, 41(4), 36-40. https://doi.org/10.3103/ S0095452707040068

Pisano, E. (1972). Observaciones Fito-ecológicas en las islas Diego Ramírez. Anales del Instituto de la Patagonia, 1-2, 161-169.

Pisano, E. (1980a). Catálogo de la flora vascular del archipiélago del Cabo de Hornos. Anales del Instituto de la Patagonia, 11, 151-189.

Pisano, E. (1980b). Distribución y características de la vegetación del Archipiélago del Cabo de Hornos. Anales del Instituto de la Patagonia, 11, 191-224.

Pisano, E., \& Schlatter, R. (1981a). Vegetación y flora de las islas Diego Ramírez (Chile).

I. Características y Relaciones de la flora vascular. Anales del Instituto de la Patagonia, 12, 183-194.

Pisano, E., \& Schlatter, R. (1981b). Vegetación y flora de las islas Diego Ramírez (Chile). II. Comunidades Vegetales vasculares. Anales del Instituto de la Patagonia, 12, 195-204.

Primack, R., Rozzi, R., Feinsinger, P., Dirzo, R., \& Massardo, F. (Eds.) (2001). Fundamentos de conservación biológica. Perspectivas latinoamericanas. México: Fondo de Cultura Económica.

Robertson, G., Moreno, C. A., Lawton, K., Arata, J., Valencia, J., \& Kirkwood, R. (2007). An estimate of the population sizes of Blackbrowed (Thalassarche melanophrys) and Grey-headed ( $T$. chrysostoma) Albatrosses breeding in the Diego Ramírez Archipelago, Chile. Emu, 107, 239-244.

Rodríguez, R., Marticorena, C., Alarcón, D., Baeza, C., Cavieres, L., Finot, V. L., Fuentes, N., Kiessling, A., Mihoc, M., Pauchard, A., Ruiz, E., Sánchez, P. \& Marticorena, A. (2018). Catálogo de las plantas vasculares de Chile. Gayana Botánica, 75, 1-430.

Rozzi, R., Charlin, R., Ippi, S., \& Dollenz, O. (2004). Cabo de Hornos: un parque nacional libre de especies exóticas en el 
confín de América. Anales del Instituto de la Patagonia, 32, 55-62.

Rozzi, R., Massardo, F., Anderson, C., Heidinger, K., \& Silander Jr, J. (2006). Ten principles for biocultural conservation at the southern tip of the Americas: the approach of the Omora Ethnobotanical Park. Ecology \& Society, 11(1), 43.

Rozzi, R., Armesto, J. J., Gutiérrez, J., Massardo, F., Likens, G., Anderson, C., Poole, A., Moses, K., Hargrove, E., Mansilla, A., Kennedy, J. H., Willson, M., Jax, K., Jones, C. G., Callicott, J. B. \& Kalin, M. T. (2012). Integrating ecology and environmental ethics: Earth stewardship in the southern end of the Americas. BioScience, 62(3), 226-236.

Rozzi, R., Massardo, F., Mansilla, A., Squeo, F. A., Barros, E., Contador, T., Frangopulos, M., Poulin, E., Rosenfeld, S., Goffinet, B., González-Weaver, C., Mackenzie, R., Crego, R.D., Viddi, F., Naretto, J., Gallardo, M.R., Jiménez, J.E., Marambio, J., Pérez, C., Rodríguez, J.P., Méndez, F., Barroso, O., Rendoll, J., Schüttler, E., Kennedy, J., Convey, P., Russell, S., Berchez, F., Sumida, P.Y.G., Rundell, P., Rozzi, A., Armesto, J., Kalin-Arroyo, M. \& Martinic, M. (2017). Parque Marino Cabo de Hornos-Diego Ramírez. Informe Técnico para la Propuesta de Creación. Programa de Conservación Biocultural Subantártica, Ediciones Universidad de Magallanes. Punta Arenas, Chile

Rozzi, R., Crego, R. D., Contador, T., Schüttler,
E., Rosenfeld, S \& Massardo, F. (2020). Un centinela para el monitoreo del cambio climático y su impacto sobre la biodiversidad en la cumbre austral de América: la nueva red de estudios socio-ecológicos a largo plazo Cabo de Hornos. Anales del Instituto de la Patagonia, 48, en revisión.

Schlatter, R., \& Riveros, G. (1987). Historia Natural del Archipiélago Diego Ramírez, Chile. Ser. Cient. INACH, 47, 87-112.

Shaw, J. D. (2005). The Reproductive Ecology of Vascular Plants on Subantarctic Macquarie Island (Tesis de Doctorado). University of Tasmania, Australia.

Squeo, F. A., Estades, C., Bahamonde, N., Cavieres, L. A., Rojas, G., Benoit, I., Parada, E., Fuentes, A., Palma, A., Solís, R., Montenegro, G., \& Torres-Mura, J. C. (2010). Revisión de la clasificación de especies en categorías de amenaza en Chile. Revista Chilena de Historia Natural, 83, 511-529.

Turner, P. A. M., Scott, J. J., \& Rozefelds, A. C. (2006). Probable long distance dispersal of Leptinella plumosa Hook. f. to Heard Island: habitat, status and discussion of its arrival. Polar Biology, 29, 160-168.

Vitousek, P. M. (1994). Beyond global warming: ecology and global change. Ecology, 75, 1861-1876.

Vitousek, P. M., D'Antonio, C. M., Loope, L. L., \& Westerbrooks, R. (1996). Biological invasions as global environmental change. American Scientist, 84, 468-478. 